\title{
WASOS: An Ontology for Modelling Traditional Knowledge of Sustainable Water Stewardship
}

\author{
Andreas Vlachidis ${ }^{1[0000-0003-0986-4430]}$ and Mark Everard ${ }^{20000-0002-0251-8482]}$ \\ ${ }^{1}$ Department of Information Studies, University College London, Gower Street, WC1E 6BT \\ London, UK \\ a.vlachidis@ucl.ac.uk \\ ${ }^{2}$ Geography and Environmental Management, University of the West of England, Coldharbour \\ Lane, Bristol BS16 1QY, UK \\ mark. everard@uwe.ac.uk
}

\begin{abstract}
Recent work and publications concerning sustainable water stewardship in Rajasthan (India) highlight how contemporary challenges are eroding traditional, communal approaches to water stewardship through mechanised extraction beyond the renewable capacities of ecosystems. Our work is focused on developing a formal ontology for modelling the knowledge of traditional water stewardship in India's drylands by capturing the key constitutional elements of regenerative methods. Our method follows an iterative evolving prototype process for delivering the first version of the Ontology for Sustainable Water Stewardship (WASOS). The ontology contains a moderate number of high-level classes and properties that represent the water management decision-making process. By making key relationships visible, we aim to support decision-making in complex catchments particularly where there are contested urban and rural claims on water.
\end{abstract}

Keywords: Ecoinformatics, Water Stewardship, Ontology, Ecosystem, India.

\section{Introduction}

The purpose of this paper is to capture the traditional water stewardship knowledge that has enabled societal progress over four-and-a-half thousand years in India's drylands. This is achieved by modelling of ontologies, in order to inform and guide water management in the face of modern challenges (population, urbanisation, industrialisation and climate change). This project aims to model the key constitutional elements of decision-making systems in terms of entities, attributes and relationships that can potentially protect or restore ecosystem processes to support human wellbeing into the longer-term future. It also serves to assess the feasibility of the use of ontologies (formal conceptual structures) in the context of water stewardship at landscape scale, with the aim of exploring the potential of ontologies for embedding sophisticated statements of environmental knowledge particular to practices and methods underpinning sustainable water stewardship. 
Recent work and publications concerning sustainable water stewardship in Rajasthan (India) highlight how contemporary challenges are eroding traditional, communal approaches to water stewardship focused on sustainable and equitable management of water resources recharged only during episodic monsoon rainfall [1]. Expertise from the contrasting disciplines of water management and ontology modelling can support the aims of systematising 'traditional wisdoms'. It is evident that bio-ontologies can enable the modelling, discovery and unified querying of multidimensional and heterogeneous environmental data resources while benefiting automatic procedures for collection, selection, annotation and indexing of data [2][3]. Ontologies have already greatly benefited the agricultural domain providing definitions for crops and crop products, agricultural management, and agricultural and environmental policy [4]. We envisage that ontologies can be similarly applied to the water management domain to formally express and integrate traditional knowledge of water stewardship, informing water management decision making process towards a sustainable form of development that makes a wise use of scarce water resources.

\section{Background}

\subsection{Background on decision-making challenges and issues in dryland India}

Groundwater is a critically important resource for Indian dryland systems. This is due to the fact that episodic monsoon rainfall occurs only in a short window of time, and the high temperatures and evaporation rates throughout the rest of the year result in substantial evaporation rapidly drying soil moisture and surface water bodies. Over millennia, rural communities have adapted innovative physical structures, community governance arrangements and water use habits to subsist in these challenging conditions [5]. However, in India in general, and in Rajasthan and adjacent Indian dryland states in particular, a pervasion of modern, mechanised technologies are driving overabstraction of groundwater well beyond natural regeneration rates [1]. Understanding the dynamics of decision-making processes around water is a pressing priority as a contributor to wiser and more sustainable management of scarce water resources.

\subsection{Background on the role of ontologies in environmental decision-making}

As ecological and environmental sciences expand their scope to larger, interdisciplinary and collaborative networks, the potential role of informatics offers increasing promise for facilitating the use and exploration of scientific data and information [6]. Ecoinformatics can deliver this potential by making evident often formerly subliminal conceptual processes and enabling generation of new knowledge, discovery, integration and analysis of biological, environmental, and socioeconomic data through the use of innovative tools and computational methods [7]. Ontologies constitute the main digital artefact in the stack of the Semantic Web (SW) technologies for the formal representation of the semantics and conceptual arrangements of data. Ontology-driven data integration can significantly improve the semantic interoperability, analysis and synthesis of data 
whereas the Web Ontology Language (OWL) is capable of modelling concepts of complex domains and enhance the interoperability of multiple sources of data [8].

The interdisciplinary characteristics of the environmental sciences and the breadth of research questions associated with the field, which can span from gene to biosphere, create the need for effective access and sharing of data and information resources. The heterogeneity and disparity of such resources is a hindrance to their discovery, integration and analysis. Collaborative systems and information sharing tools should be capable of enabling scientists to access, trust and understand the shared load of information without inflicting biases or misconceptions.

Several examples in the domain of environmental studies have employed ontologies for describing the semantics of observational data sets. The InWaterSense project has developed a set of ontologies (i.e. core, regulation and polluters) for modelling observational data types of water quality, regulations and other water domain knowledge (e.g., water-relevant contaminants, bodies of water, etc.) [9]. The Science Environment for Ecological Knowledge (SEEK) has developed the Extensible Observation Ontology (OBOE) [10] as a formal and generic conceptual framework for describing the semantics of observational data sets based on the concepts of Observation, Measurement, Unit, Characteristic, and (Ecological) Entity. The OntoAgroHidro ontology of the Embrapa's research network represents knowledge about the impacts of climatic changes and agricultural activities on water resources [6]. The Consortium of Universities for the Advancement of Hydrologic Science has developed the Cuahsi Ontology [11] for describing hydrologic concepts, whereas the Semantic Web for Earth and Environmental Terminology (SWEET) developed by NASA Jet Propulsion Laboratories contains over 6,000 science concepts organized in 200 ontologies represented in OWL [12].

\section{$3 \quad$ Method}

An abundance of ontology engineering methodologies is available in the scientific literature. Early attempts to develop an ontology engineering methodology date as back to the mid-1990s. They articulate several steps as important for building an ontology, including identification of the ontology's purpose, ontology coding, integration of existing ontologies, ontology evaluation and ontology documentation. The Methontology approach [13] is an early example demonstrating the added value brought in the ontology development process by an iterative, evolving prototype approach.

Our work is focused on capturing the key constitutional elements of regenerative water stewardship methods in terms of entities, attributes and relationships. The first version of the WASOS ontology is aimed at formally expressing the key constitutional elements of decision-making systems and water stewardship governance in Rajasthan with a direct effect on water sustainability, rather than attempting to capture all relevant entities of the water management domain. Therefore, concepts and relationships relating to water quality, environmental and ecological domain were not the primary focus of the ontology design. The abundance of ontology models for capturing ecological and environmental knowledge, as already discussed, represent a significant resource and 
channel for directing and informing further tasks of ontology reuse and alignment, which will be considered and addressed by the future versions of the ontology.

Our work is mainly informed by the Methontology approach and is primarily focused on the development-oriented tasks of specification, conceptualisation and implementation. The design of the ontology was driven by a team of three experts (information scientist, environmentalist and a water stewardship expert) who regularly met and discussed the aims and scope of entities and relationships.

\section{The WASOS Ontology}

The alpha version WASOS ${ }^{1}$ ontology contains a moderate number of high-level classes and properties which were concluded after a several design iterations and discussion between experts. It contains 44 class declarations, 14 object property declarations and 202 axioms in total. This alpha version is a 'work in progress' that sets the foundations of an ontology aimed at capturing the knowledge of traditional water stewardship methods. Therefore, the range and scope of the entities as discussed below constitute an initial understanding of the domain which, to the best of our knowledge, has not been modelled before using formal languages.

\subsection{Ontology Classes}

Activity: the class comprises of actions carried out by Person that result to a permanent change of the state of a tangible (e.g. Physiographic Feature) or intangible (e.g. Policy) matter. An Activity has a temporal duration which can be short-lived or ongoing. Subclasses are; Water Harvesting, Issue Right, Remove Right, Issue Penalty.

Administration Area: the class comprises of instances of geographical areas of political administration connected to local or national government. Such areas are administer by instances of Governance Unit which in their turn are composed of instances of Person. Subclasses are; Block, District, Stage, Village.

Financial Resources: the class comprises of resources of monetary value which are made available to support actions relating to water stewardship. Subclasses are; Government Fund, International Donation.

Governance Unit: the class comprises of all different types of instances of governance including elected or appointed units of local or national level that can take the form of councils, boards, and offices. Subclasses are; Block Development Office, Block Panchayat, Gram Panchayat, Informal Governance Unit, Vidhan Parishad, Zilla Parishad.

Measurement: This class comprises of measurement actions relevant to water stewardship which determine properties and values by a systematic procedure.

Person: the class comprises of instances of real persons which relate, contribute, participate or can be broadly associated with water stewardship. Subclass are; Villager,

\footnotetext{
1 The alpha version of the WASOS ontology is available from the Github repository at https://github.com/avlachid/WASOS
} 
Worker, Government Unit Member, Head of Administration, Block Development Officer, CEO, Pradhan, Sarpanch

Physiographic Feature: the class comprises of real world instances of identifiable features of the natural world. The class contains two main subclasses, Water body and Land Feature which in turn contain the subclasses River, Pond, Pool, Nullah (ravine) and Mountain, Field, Forest, respectively.

Policy: the class comprises of instances of statements, plans and procedures that can take the form of formal documentation. Sub classes are; Agreement, Civil Sanction, Consent, Requirement.

\subsection{Ontology Properties}

The ontology contains a small set of object properties focused on modelling the relationships between the classes Person, Administrative Area and Governance Unit Person. The properties identify relationships in connection to the composition of administrative areas, governance of such areas and member participation to governance. Larger administrative areas are composed of smaller administrative areas, whereas each area is governed by a governance unit which is made of members and is chaired by a head of administration. Figure 1 presents the object properties and the respective domain and range classes, including subclass relationships.

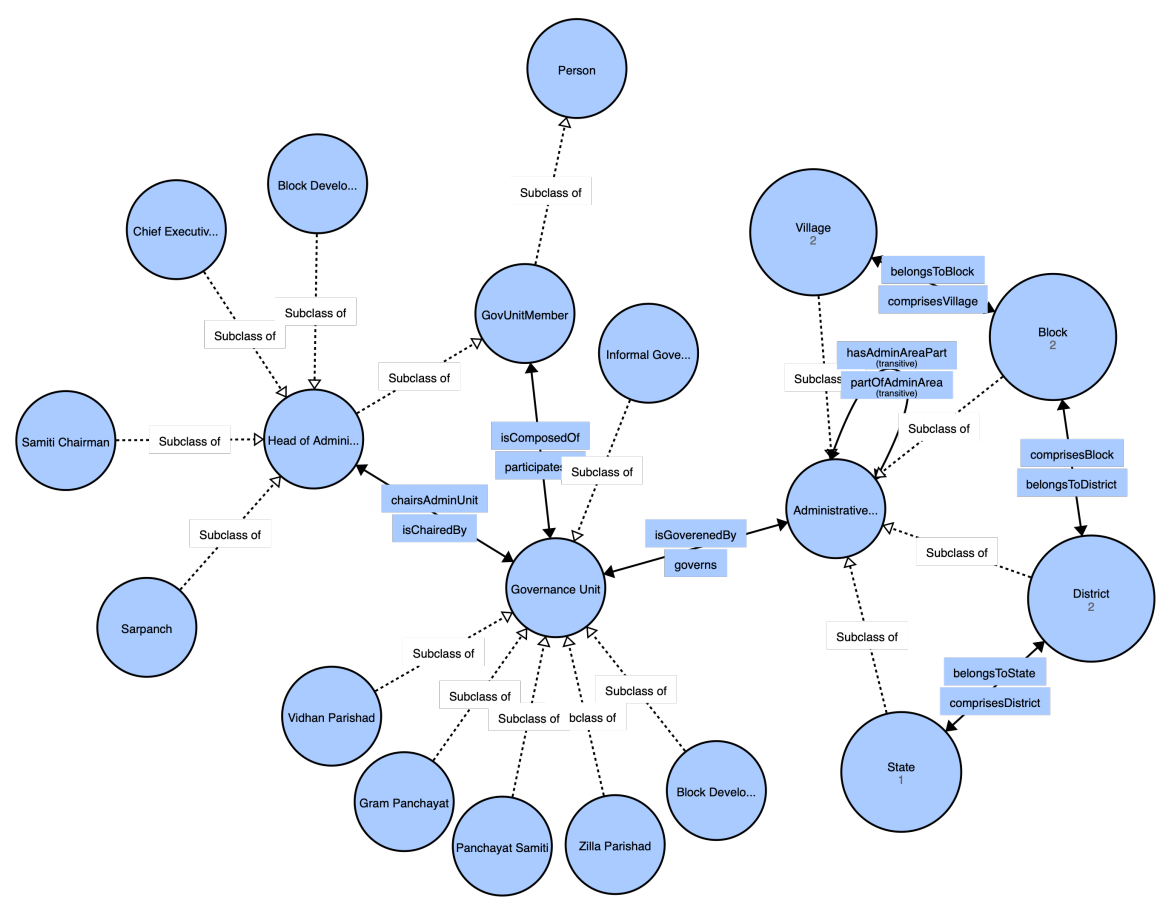

Fig. 1. WASOS ontology: a graph of classes and properties focused on modelling the relationships between Person, Administrative Area and Governance Unit. Dotted lines represent subclass relations, solid black lines represent object properties. 


\section{Discussion}

Key success criteria for regeneration of water resources supporting socio-economic wellbeing in rural Rajasthan included a fully systemic approach to water stewardship, in which decision-making and ongoing management implements water management solutions that work with natural catchment processes supporting landscape and water use on a sustainable basis [1]. Recognition of these success criteria is essential if reversal of former declines in linked socio-ecological systems is to influence water resource decisions across broader and more complex catchments subject to competing rural and urban demands. It is therefore important that the decision-making apparatus, including institutions and individual, decision-making frameworks and key criteria are understood and transparently articulated, hence the value of their representation in the form of an ontology.

Key elements represented in the ontology presented here include administrative levels (village, block, district and state), how they relate in terms of input from environmental criteria (such as water bodies) and how they interact through decision-making forums. Ensuring that this network of institutions and decision-making links is represented in decisions can support more sustainable and equitable policy and practical outcomes, and may be transferrable with adaptation more widely across India and other dryland global regions. An integrated approach to catchment management is essential not merely for addressing directly water-related goals but in support of linked goals such as the contribution of sustainable water systems to food security and health [14].

\section{Conclusions and Future Work}

The ontology delivers a first (alpha) version of formal semantics dedicated to capturing the knowledge of traditional water stewardship methods whilst providing a transparent representation of power and decision-relevant interactions, which may be applied to environmental decision-making situations. Using ontologies for representing the water management decision-making process and by making key relationships visible, we aim to support decision-making in complex catchments particularly where there are contested urban and rural claims on water, both in India (Rajasthan) and beyond. The current version constitutes a proposition to further discussion and development of a more comprehensive ontology of the domain of traditional water stewardship.

We envisage that further development of our preliminary approach could support sustainable management of water in arid and semi-arid environments, promoting attainment of the UN Sustainable Development Goals. Future steps include the expansion of the ontology to cover the range of entities relating to the domain of water stewardship such as buildings and infrastructure, subclasses of physiographic features, events and activities and the definition of a comprehensive set of object properties that implement the relationships between ontology classes. In addition, we are planning to incorporate into the ontology elements of the Agrovoc ${ }^{2}$ thesaurus in order to provide vocabulary specialisations and to investigate the possibilities of re-using classes from other domain

\footnotetext{
${ }^{2}$ http://aims.fao.org/vest-registry/vocabularies/agrovoc
} 
ontologies identified in section 2.2. We are also planning for a thorough evaluation of the ontology in real case study by means of quantitative and qualitative metrics, such as accuracy, completeness, adaptivity using established evaluation methods [15].

\section{References}

1. Everard, M: Community-based groundwater and ecosystem restoration in semi-arid north Rajasthan (1): socio-economic progress and lessons for groundwater-dependent areas. Ecosystem Services 16, 125-135 (2015).

2. Lokers, R., Van Randen, Y., Knapen, R., Gaubitzer, S., Zudin, S. and Janssen, S. Improving access to big data in agriculture and forestry using semantic technologies. In Research Conference on Metadata and Semantics Research, 369-380 (2015).

3. Keet, C.M.: Ontology design parameters for aligning agri-informatics with the Semantic Web. In Research Conference on Metadata and Semantic Research, 239-244 (2009)

4. Athanasiadis, I.N., Rizzoli, A.E., Janssen, S., Andersen, E. and Villa, F.: Ontology for seamless integration of agricultural data and models. In Research Conference on Metadata and Semantic Research, 282-293 (2009).

5. Pandey, D.N., Gupta, A.K., Anderson, D.M.: Rainwater harvesting as an adaptation to climate change. Current Science, 85(1), 46-59 (2003).

6. Bonacin, R., Nabuco, O.F., Pierozzi Junior, I.: Ontology models of the impacts of agriculture and climate changes on water resources: Scenarios on interoperability and information recovery. Future Generation Computer Systems 54, 423-434 (2016).

7. Michener, W.K., Jones, M.B.: Ecoinformatics: supporting ecology as a data-intensive science. Trends in Ecology \& Evolution 27, 85-93 (2012).

8. Ding, L., Michaelis, J., McCusker, J., McGuinness D.L.,: Linked provenance data: a semantic Web-based approach to interoperable workflow traces, Future Genereration Computer. Systems. 27, 797-805 (2011).

9. Ahmedi, L., Jajaga, E., Ahmedi, F.: An Ontology Framework for Water Quality Management. In: Corcho, Ó., Henson, C.A., Barnaghi, P.M. (eds.) SSN@ISWC, pp. 35-50 (2013)

10. Madin, J., Bowers, S., Schildhauer, M., Krivov, S., Pennington, D., Villa, F.: An ontology for describing and synthesizing ecological observation data. Ecological informatics 2, 279296 (2007).

11. Tarboton, D.G., Maidment, D., Zaslavsky, I., Ames, D., Goodall, J., Hooper, R.P, Horsburgh, J., Valentine, D., Whiteaker, T., Schreuders, K.: Data interoperability in the hydrologic sciences, the cuahsi hydrologic information system, in: Proceedings of the Environmental Information Management Conference, Santa Barbara, CA, pp. 132-137 (2011).

12. Raskin, R.G, Pan, M.J, Knowledge representation in the semantic web for Earth and environmental terminology (SWEET), Computers and Geoscience. 31 (9), 1119-1125 (2005)

13. Fernández-López, M., Gómez-Pérez, A., Juristo, N.: METHONTOLOGY: From Ontological Art Towards Ontological Engineering, In: Proceedings of the Ontological Engineering AAAI-97 Spring Symposium Series. Presented at the AAAI-97 Spring Symposium Series, Facultad de Informática (UPM), Stanford University, EEUU. (1997)

14. Everard, M: Repurposing business around the meeting of human needs. Environmental Scientist, September 2017, 40-45 (2017)

15. Hlomani, H. and Stacey, D.: Approaches, methods, metrics, measures, and subjectivity in ontology evaluation: A survey. Semantic Web Journal, 1(5), 1-11 (2014). 